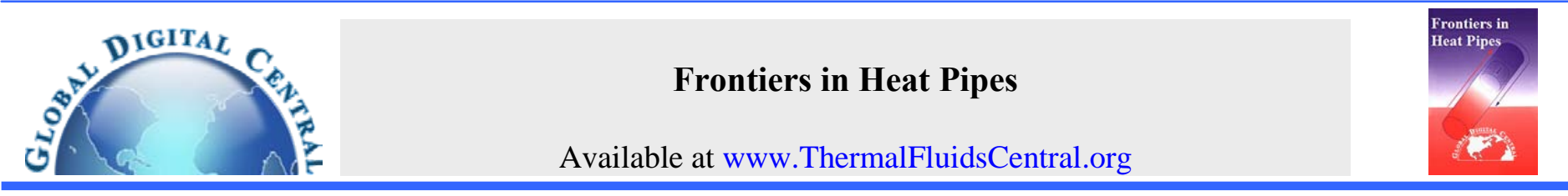

\title{
THEORETICAL STUDY AND REVIEW ON THE OPERATIONAL LIMITATIONS DUE TO VAPOUR FLOW IN HEAT PIPES
}

\author{
Rémi Bertossi, Cyril Romestant, Vincent Ayel*, Yves Bertin \\ Institut Pprime, CNRS - ENSMA - Université de Poitiers, UPR 3346 \\ Département Fluide, Thermique et Combustion \\ 1, avenue Clément Ader 86961 Futuroscope Chasseneuil Cedex, FRANCE
}

\begin{abstract}
Vapour flow in heat pipes is subject to two operating limitations that can restrict the performances of such technologies. The first one is the viscous limitation: it occurs when the operating temperature is closed to the triple point and the pressure slope on the saturation curve, related to the temperature glide, is not large enough to counterbalance the viscous pressure losses of the vapour flow. The second one is the sonic limitation: it can appear when the vapour velocity becomes high so that it reaches the speed of sound. This paper presents a detailed description of the physical phenomena which take place in these two types of limitations. Some analytical expressions of maximal heat flux induced by the latter are also proposed.
\end{abstract}

Keywords: viscous limitation, sonic limitation, analytical study, vapour, heat-pipes

\section{INTRODUCTION}

Heat pipes working are very dependent on their operational limitations. Indeed, maximal performances are limited by the operating conditions of the device. Five types of limitations are classically defined. Fig. 1 represents the operating domain of heat pipes as a function of the different limitations that can come into play (Faghri, 1995). The latter are notably very dependent on the thermophysical properties of the working fluid; for example, for working temperatures closed to the triple point or to the critical one, the performances can be seriously deteriorated. Besides, limitations are also directly linked to the characteristics of the fluid flow.

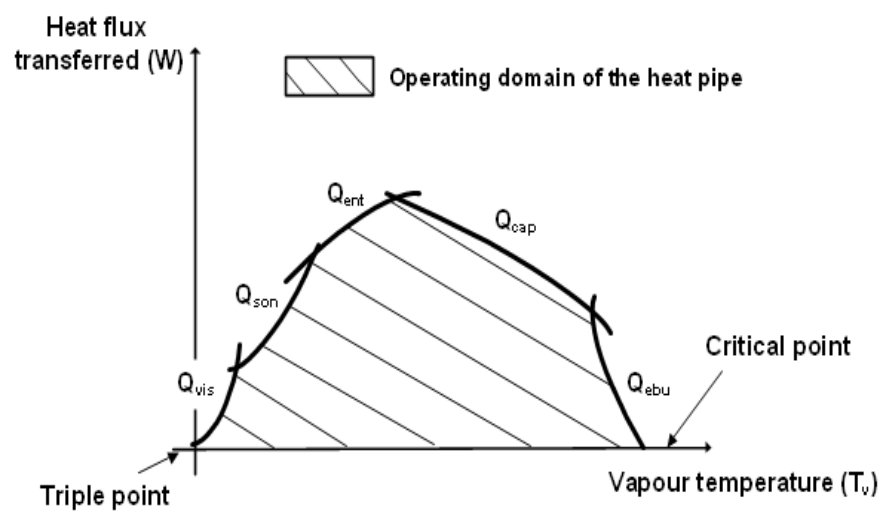

Fig. 1 Operating limitations interfering in the heat pipe working

The operating limitations can be divided into two main categories: the first ones linked to the liquid flow $\left(Q_{e n t}, Q_{c a p}, Q_{b o i l}\right.$ on Fig. 1) and the other ones linked to the vapour flow $\left(Q_{v i s}, Q_{\text {son }}\right.$ on Fig. 1). For the liquid flow, one has:

- The entrainment limitation, $Q_{e n t}$, that can occur when the vapour velocity is very high so that liquid particles can be pulled out from the liquid flow which is consequently altered and not able to carry out properly heat flow from the condenser into the evaporator.

- The capillary limitation, $Q_{c a p}$, that is linked to the maximal pumping capacity of the heat pipe that counterbalances the pressure losses both in vapour and liquid flows. The latter depends on the heat pipe filling, the porous characteristics, the working fluid and the operating conditions. The capillary limitation represents the main limitation for capillary pumping heat pipes. It is mainly relative to the liquid flow because the viscous pressure losses are generally more important in the liquid flow than in the vapour flow.

- the boiling limitation, $Q_{\text {boil }}$, that can be attained when the superheat at the wall becomes too important in the evaporator, then triggering nucleation at the liquid wall and altering liquid flow.

The two other limitations, $Q_{\text {vis }}$ and $Q_{\text {son }}$, strictly concern the vapour flow. They correspond to the viscous and the sonic limitations, respectively. The first one occurs for flows whose temperatures are much closed to the triple point (Fig. 1). The second one corresponds to vapour flow velocities which have attained the speed of sound; both are generally preponderant for low density of the vapour phase. In macro heat pipes, the vapour flow is not directly linked to the liquid flow. Indeed, it is generally admitted that the variations of the liquid film thickness along the heat pipe do not affect the section of the vapour phase. Consequently, determining the maximal transfer capacity of heat pipes, for specific operational limitations, only taking vapour flow into account, is quite possible.

\footnotetext{
*Corresponding author.Email: vincent.ayel@ensma.fr
} 
This paper focuses on these two types of limitations concerning the vapour flow and presents a description of the physical phenomena interfering in this flow for different operating conditions. Analytical expressions of maximal heat flows induced by these limitations are also proposed. Note that all fluids thermophysical properties have been taken from the NIST Scientific and Technical Database (except for the Sodium)

\section{VISCOUS LIMITATION}

\subsection{Theoretical analysis}

When the operating temperature is close to the triple point, the maximal pressure gradient of vapour flow between the evaporator and the condenser in heat pipes tends to greatly decrease; this behaviour can be explained thanks to Clausius-Clapeyron equation (Eq. (1)), due to the decrease of the pressure slope $d P_{v} / d T_{v}$ as the saturated temperature decreases:

$d P_{v}=\frac{\rho_{v} h_{l v}}{T_{v}} d T_{v}$

This relation can be used here because the vapour is assumed to be at saturation state all along the heat pipe. So, when vapour density is very low (typically, inferior to $10^{-2} \mathrm{~kg} . \mathrm{m}^{-3}$ ), a given temperature gradient generates a very low pressure gradient. To illustrate this phenomenon, Table 1 gathers some typical numerical values of the derivatives of the saturation curve as functions of temperatures, for four classical working fluids.

Table 1 Numerical values of the derivative of the saturation curve $\left(d P_{v} / d T_{v}\right)$ for different fluids.

\begin{tabular}{|c|c|c|c|c|c|}
\hline Fluid & $\begin{array}{c}T_{v} \\
(\mathrm{~K}) \\
\end{array}$ & $\begin{array}{c}\rho_{v} h_{l v} / T_{v} \\
\left(\mathrm{~Pa} \mathrm{~K}^{-1}\right) \\
\end{array}$ & Fluid & $\begin{array}{c}T_{v} \\
(\mathrm{~K}) \\
\end{array}$ & $\begin{array}{c}\rho_{v} h_{l v} / T_{v} \\
\left(\mathrm{~Pa} \mathrm{~K}^{-1}\right) \\
\end{array}$ \\
\hline \multirow{4}{*}{ Water } & 273.16 & 44.4 & \multirow{4}{*}{ Sodium } & 600 & 0.16 \\
\hline & 278.15 & 60.8 & & 700 & 2.45 \\
\hline & 283.15 & 82.2 & & 800 & 17.3 \\
\hline & 293.15 & 144.7 & & 900 & 75.7 \\
\hline \multirow{4}{*}{ Mercury } & 273.15 & $2.7 \times 10^{-3}$ & \multirow{4}{*}{ Lithium } & 500 & $0.81 \times 10^{-9}$ \\
\hline & 283.15 & $6.5 \times 10^{-3}$ & & 600 & $0.27 \times 10^{-6}$ \\
\hline & 313.15 & 0.065 & & 700 & $1.58 \times 10^{-3}$ \\
\hline & 373.15 & 1.96 & & 800 & 0.032 \\
\hline
\end{tabular}

Triple Point: Water 273.16 K, Sodium 371 K, Mercury 234.3K, Lithium $453.7 \mathrm{~K}$

In Table 1, values written in bold characters correspond to very low values of the derivative of the saturation curve and so, to situations where the viscous limitation can preferentially occur. Water density at temperatures close to the triple point is low, but remains high enough to ensure a significant pressure gradient. On the contrary, for liquid metals, the value is so little that the pressure drop is not sufficient to counterbalance viscous pressure losses and the viscous limitation can be quickly attained. Consequently, heat transfer capability of liquid metal heat pipes can be highly deteriorated at temperatures closed to the triple point.

In order to completely determine the flow pressure drop, NavierStokes equations should be completely solved since the vapour flow is a priori at least two-dimensional because of parietal injection of heat flux.

As far as we know, as these complicated numerical calculations are still not performed in the literature, an approximated solution, taking into account the main physical phenomena, can be developed in order to have an acceptable order of magnitude of the viscous limitation.

In a first extent, a numerical approximated solution is presented. Starting from this approximation, an analytical approximated solution is then proposed to easily obtain an evaluation of the maximal heat flux corresponding to this viscous limitation. Note that, in this study, the vapour flow characteristics are considered not to be those of a rarefied gas, considering flow regimes with Knudsen number much lower than unity.

\subsection{Approximated numerical solution}

In this section, one considers heat pipe with a circular section and with lengths of evaporator, adiabatic zone and condenser important compared to the inner diameter of the vapour section $\left(L_{i} / D_{v}>10\right.$ with $i$ $=e, a$ or $c$, for evaporator, adiabatic zone and condenser, respectively). Besides, boundary conditions are assumed to be symmetrical all around the heat pipe. For these reasons, pressure is here considered only as a function of the abscissa $z$; radial gradients are neglected (Fig. 2). As viscous limitation is defined as regards to relative low pressure gradient and given the cylindrical geometry of the problem, the hypothesis of a Poiseuille-type laminar and incompressible flow is adopted here.
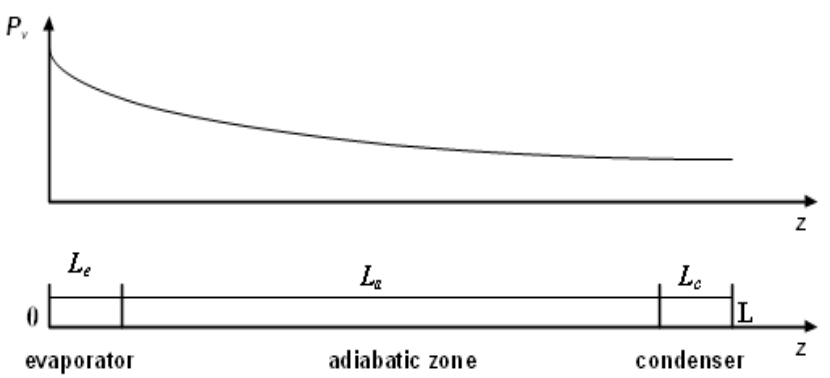

Fig. 2 Evolution of the vapour pressure field along the heat pipe.

In this case, the pressure losses along the heat pipe are given by the following relation:

$$
\frac{d P_{v}(z)}{d z}=-\frac{32 \mu_{v}(z)}{D_{v}^{2}} \bar{U}_{v}(z)
$$

with $\bar{U}_{v}(z)$ the average vapour velocity in a given section of the heat pipe and $D_{v}$ the diameter of the vapour section, assumed here to be constant.

Let us notice that the vapour dynamic viscosity $\mu_{v}(z)$ is dependent on both pressure and temperature, so it is constant in a cross-section of the heat pipe but varies along the vapour flow, i.e. along abscissa $z$. Besides, mass and heat flux carried out by the vapour flow in a section of the heat pipe are linked by the classical relation (3):

$Q(z)=\dot{m}(z) h_{l v}$

Here, the latent heat of phase change $h_{l v}$ is considered constant because, for temperatures closed to the triple point, it does not vary much. The mass flow in a section is obviously given by the following equation:

$\dot{m}(z)=\rho_{v}(z) \bar{U}_{v}(z) S_{v}$

Like viscosity, vapour density is assumed constant in a crosssection and is evaluated at saturated temperature defined by the pressure in the considered section. In Eq. (4), $S_{v}$ corresponds to the transversal cross-section of the vapour flow $\left(S_{v}=\pi r_{v}{ }^{2}\right)$. Then, the average vapour velocity in a given section can be obtained by: 


$$
\bar{U}_{v}(z)=\frac{Q(z)}{h_{l v} \pi r_{v}^{2} \rho_{v}(z)}
$$

Combining equations (2) and (5), one obtains the final expression for vapour viscous pressure losses:

$$
\frac{d P_{v}(z)}{d z}=-\frac{8}{\pi r_{v}^{4} h_{l v}} \frac{\mu_{v}(z) Q(z)}{\rho_{v}(z)}
$$

$Q(z)$ can be easily calculated along the heat pipe integrating the parietal heat flux density both at evaporator and condenser. Besides, if the vapour is considered at saturation state all along the heat pipe, the dynamic viscosity and the density can be expressed only as functions of pressure. But, as seen before, the pressure is a function of $z$; so, it is possible to integrate Eq. (6) in order to obtain the pressure profile.

The viscous limitation $Q_{v i s}$ is chosen as the maximal heat flux that can be carried out by the vapour flow for a given temperature, taken in the middle of the adiabatic zone. The latter temperature corresponds to the reference operating conditions in heat pipes. The boundary condition used for this limitation is the saturation pressure at the triple point at the end of the condenser (at $z=L$ on Fig. 2), which corresponds to the maximal pressure gradient between the adiabatic zone and the extremity of the condenser. Consequently, for a given heat flux density at the condenser, the pressure profile can be calculated, and, particularly, the pressure in the middle of the adiabatic zone can be determined. The saturated temperature corresponding to this pressure is used to define the viscous limitation point. So, by varying the condensation heat flux, the curve $Q_{v i s}\left(T_{v}\right)$ can be drawn and the viscous limitation characterized.

This numerical solution imposes the resolution of Eq. (6) but the value of $Q_{v i s}$ cannot be directly obtained. That is why, in the following section, thanks to simplifying hypotheses, one proposes here an analytical expression for the viscous limitation $Q_{\text {vis. }}$.

\subsection{Approximated analytical solution}

To obtain an analytical expression of the viscous limitation $Q_{\text {vis }}$, Busse (1973) has made a strong hypothesis, also used by Faghri (1995): the vapour flow is assumed to be isothermal. This hypothesis seems to be arguable given the evolution of the saturation curve (Table 1). However, if the latter is retained, in first approximation, ideal gas law gives, between two cross-sections:

$\frac{P_{v}(z)}{\rho_{v}(z)}=\frac{P_{v, 0}}{\rho_{v, 0}}=r_{g} T_{v, 0}$

where the subscript "0" refers to the section at the evaporator cap $(z=0$ on Fig. 2).

If variations of the vapour dynamic viscosity can be neglected compared to those of vapour density along the heat pipe, with equations (6) and (7), one obtains the following equation with separate variables:

$P_{v}(z) \frac{d P_{v}(z)}{d z}=-\frac{8 \mu_{v}}{\pi r_{v}^{4} h_{l v}} \frac{P_{v, 0} Q(z)}{\rho_{v, 0}}$

Integrating relation (8) between $z=0$ and $L$, the following equation is obtained:

$P_{v, 0}^{2}-P_{v, L}^{2}=\frac{16 \mu_{v}}{\pi r_{v}^{4} h_{l v}} \frac{P_{v, 0}}{\rho_{v, 0}} \int_{0}^{L} Q(z) d z$

Let us define $Q_{v i s}$ as the maximal value of $Q(z)$, i.e., for the overall heat flux transferred along the heat pipe (in the adiabatic zone), the effective length of the heat pipe can be defined as:
$L_{\text {eff }}=\frac{1}{Q_{\text {vis }}} \int_{0}^{L} Q(z) d z$

Then, Eq. (9) gives:

$Q_{v i s}=\frac{\pi r_{v}^{4} h_{l v}}{16 \mu_{v} L_{e f f}}\left(1-\frac{P_{v, L}^{2}}{P_{v, 0}^{2}}\right) \rho_{v, 0} P_{v, 0}$

This expression does not involve the adiabatic temperature or pressure, but the conditions at both extremities of the heat pipe (at $z=0$ and $z=L$ ). In accordance with the preceding analysis led in section 2.2, Eq. (9) can also be integrated between the centre of the adiabatic zone (at $z_{\text {adia }}=L_{e}+L_{a} / 2$ ) and the end of the condenser (at $z=L$ ). Indeed, the upstream flow is out of interest given the fact that the reference temperature is defined in the adiabatic zone, given as reference temperature and pressure. Consequently, one obtains:

$Q_{v i s}=\frac{\pi r_{v}^{4} h_{l v}}{16 \mu_{v} L_{e f f}^{\prime}}\left(1-\frac{P_{v, L}^{2}}{P_{v, \mathrm{z}_{\text {adia }}}^{2}}\right) \rho_{v, \mathrm{z}_{\text {adia }}} P_{v, \mathrm{z}_{\text {adia }}}$

with: $L_{\text {eff }}^{\prime}=\frac{1}{Q_{\text {vis }}} \int_{z_{\text {adia }}}^{L} Q(z) d z$ and $z_{\text {adia }}=L_{e}+L_{d} / 2$.

It has been said that the flow characteristics at the end of the condenser (at $z=L$ ) are equal to those at the triple point TP. Finally, the viscous limitation is expressed as:

$Q_{v i s}=\frac{\pi r_{v}^{4} h_{l v, z_{\text {adia }}}}{16 \mu_{v, z_{\text {adia }}} L_{\text {eff }}^{\prime}}\left(1-\frac{P_{v, T P}^{2}}{P_{v, z_{\text {adia }}}^{2}}\right) \rho_{v, z_{\text {adia }}} P_{v, z_{\text {adia }}}$

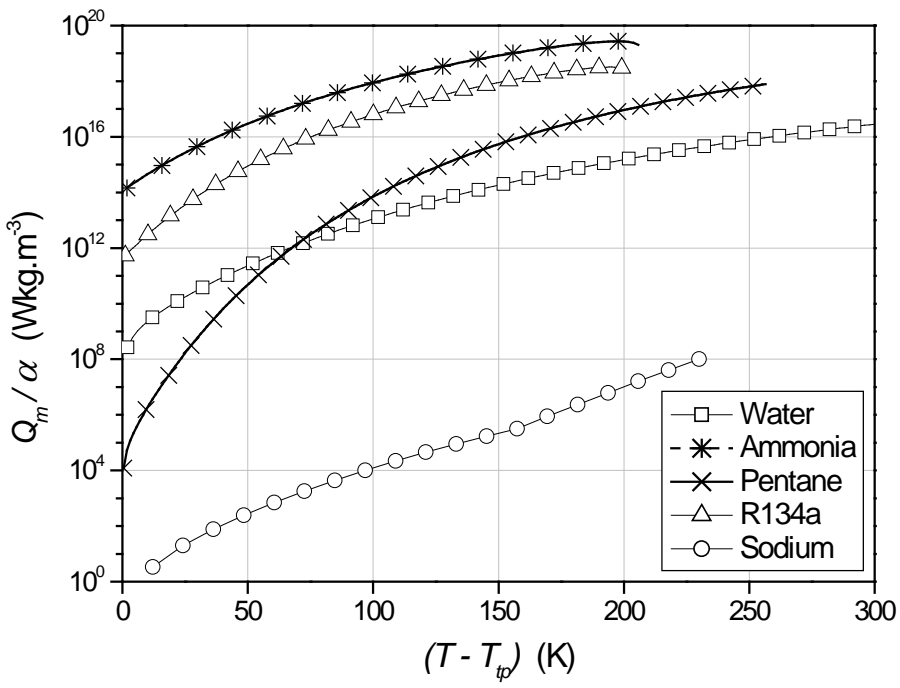

Fig. 3 Evolution of $Q_{v i s} / \alpha$ as a function of $\left(T-T_{T P}\right)$ for five fluids.

This formulation gives a very simple expression of the viscous limitation. However, given the hypothesis of an isothermal flow, it should be taken with some caution. Let define $\alpha$ as a constant geometrical parameter in Eq. (13) : $\alpha=\pi r_{v}{ }^{4} / 16 L^{\prime}{ }_{\text {eff. On Fig. 3, the }}$ evolution of $Q_{v i s} / \alpha$ is represented as a function of $\left(T-T_{T P}\right)$ for five working fluids, $T_{T P}$ being the temperature at the triple point. One can notice that the evolutions are quite different according to the fluids, with several orders of magnitude differentiating the ones to the others. As vapour metals are most often subject to this kind of limit, sodium is obviously here the fluid most affected by the viscous limit. The value of 
$Q_{v i s} / \alpha$ is at least four orders of magnitude lower than the four other fluids! Except sodium, pentane seems to be the less performing fluid for temperatures close to the triple point. Besides, as seen previously, for all fluids, the viscous limitation is minimal for the lowest temperatures.

\section{SONIC LIMITATION}

\subsection{Theoretical analysis}

\section{Sonic limitation analytical expression}

On Fig. 1, the sonic limitation appears generally after the viscous limitation with increasing temperatures, close to the triple point. This study mainly concerns the metal fluid heat pipes with very low vapour density. It occurs when vapour density is low so that its velocity reaches the speed of sound. A shock wave can then be generated and block the vapour flow, thereby preventing transmission of the heat flux from the evaporator into the condenser. Compressibility effects and pressure losses have also to be taken into account. In this paper, elementary physical hypotheses will be used to study the different possible vapour flow behaviours; a model for vapour flow will subsequently be adopted and used to obtain a simple expression of sonic limitation. Quantitative comparisons with experimental data will also be performed.

As already presented by Bertossi et al. (2011), let us assume the vapour flow in a cylindrical heat pipe to be $1 \mathrm{D}$; the maximal heat flux, attained when vapour velocity reaches the speed of sound $\left(U_{s}=\sqrt{\gamma_{g} T_{v}}\right)$, can be expressed as:

$Q_{\text {son }}=\rho_{v} U_{s} \frac{\pi}{4} D_{v}^{2} h_{l v}$

The reference temperature $T_{v, a}$ being taken in the adiabatic zone "a”, one easily obtains the following expression:

$Q_{\text {son }}=\frac{\pi}{4} D_{v}^{2} h_{l v, a} \rho_{v, a} \sqrt{r_{g} T_{v, a}}$

In Eq. (15), in order to obtain the thermodynamic state in the adiabatic zone "a", one needs to use three equations for determining the three unknown parameters: $T_{v, a}, P_{v, a}$ and $\rho_{v, a}$. First of all, in most studies concerning heat pipes, vapour is assumed to be an ideal gas (Deverall et al., 1970; Faghri, 1995; Peterson, 1994). Consequently, for a reversible and adiabatic flow, one may suppose here that the vapour phase, at the evaporator, follows the behaviour of an ideal gas: $P_{v} / \rho_{v}=r_{g} T_{v}$.

Then, as regards the variations in pressure between the beginning of the evaporator and the adiabatic zone, one may consider a momentum balance between the two parts. Differentiating mass flow rate ( $\left.\dot{m}_{v}=\rho_{v} \bar{U}_{v} S_{v}\right)$ gives:

$\frac{d \dot{m}_{v}}{\dot{m}_{v}}=\frac{d \rho_{v}}{\rho_{v}}+\frac{d \bar{U}_{v}}{\bar{U}_{v}}+\frac{d S_{v}}{S_{v}}$

Vapour density $\rho_{v}$ and velocity $\bar{U}_{v}$ are classically linked by a thermodynamic relation. Consequently, evolution of the couple $\left(\rho_{v}, \bar{U}_{v}\right)$ is a function of the variations of the section and of the mass flow rate. Considering evolution of $\left(\rho_{v}, \bar{U}_{v}\right)$ with respect to Eq. (16), an increase of relative mass flow induces an equivalent decrease of the section. So, vapour flow in constant section of a pipe with matter injection $\left(d S_{v}=0\right.$, $d \dot{m}_{v}>0$ ) shows exactly the same influence than in case of constant mass flow rate in convergent nozzle $\left(d S_{v}<0, d \dot{m}_{v}=0\right)$. This analogy is illustrated on Fig. 4. In the same way, vapour flow with condensation and no variation of section is comparable to constant mass flow with increasing section (Deverall et al., 1970).

For isentropic ideal gas flow, Candel (1990) has shown that, when the speed of sound is reached in a nozzle throat, speed and mass flow rate attain a maximal value. In the same way, in heat pipes, the sonic limitation corresponds to the heat flux transferred when maximal mass flow is attained at the end of evaporator, leading us to consider that it is when the vapour flow reaches the speed of sound.

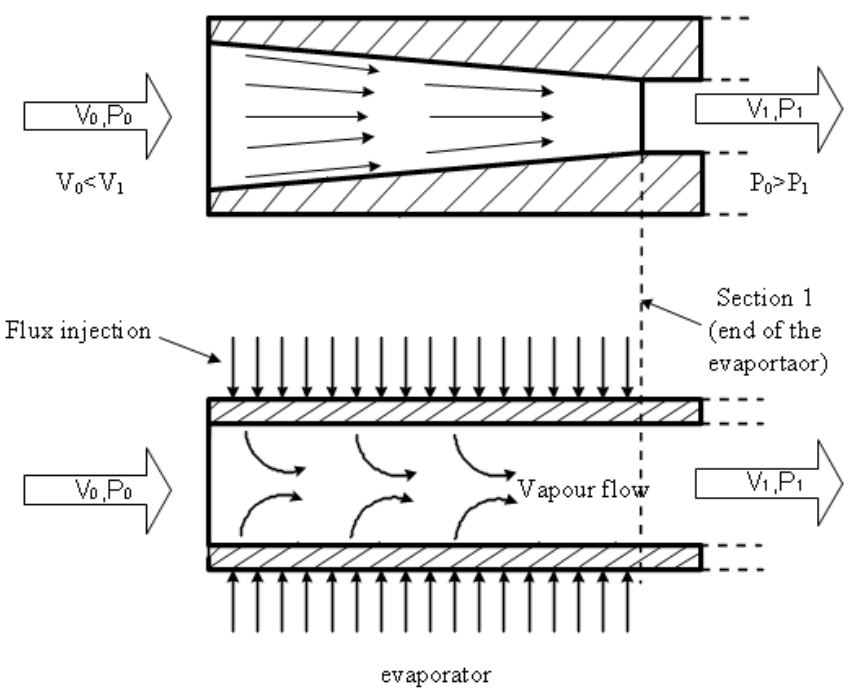

Fig. 4 Analogy of incompressible vapour flow in a heat pipe with convergent nozzle.

For a 1D vapour flow, neglecting the viscous forces, a momentum balance for the whole evaporator (with a constant section) gives: $P_{v, 0}=P_{v, 1}+\rho_{v, 1} V_{v, 1}^{2}$. In the previous equation, " 0 " refers to the entrance of the evaporator $(z=0)$ with a velocity equal to zero, and " 1 " refers to the "sonic throat" with maximal velocity (at the inlet of the adiabatic zone). Expressing vapour velocity as a function of Mach number $\mathrm{Ma}$ and considering ideal gas law, the momentum balance gives:

$\frac{P_{v, 0}}{P_{v, 1}}=1+\gamma M a_{1}^{2}$

If sonic limitation occurs when $V_{v, 1}$ equals the speed of sound at the end of the evaporator $\left(M a_{1}=1\right)$, one has:

$\frac{P_{v, 0}}{P_{v, 1}}=1+\gamma$

Finally, the last equation needed to solve the system comes from the assumption that one will take concerning the temperature evolution in the vapour flow. In the following section, one will consider an isothermal flow, an isentropic flow and, finally, a flow following the saturation state.

\section{Experimental review on sonic limitation}

Unfortunately, only few experimental studies have been laid on sonic limitation. In a previous experimental work performed by Gagneux (1979) on a sodium heat pipe, one can notice in Fig. 5 that the reference temperature has to be defined precisely when ploting operating limitation curves. Indeed, Fig. 5 shows two separated curves obtained with same experimental data: one obtained with $T_{A}$ as the reference temperature (adiabatic temperature), while the other is obtained with $T_{0}$ as the reference temperature (temperature at the beginning of the evaporator). The location of the thermal sensors for $T_{0}$ and $T_{A}$ are shown on the scheme of Fig. 5.

Taking these observations as a starting point, it appears that the definition of the adiabatic temperature $T_{v, a}$ taken as reference temperature in Eq. (15) is very important for the sonic limitation evaluation. So, two classical approaches, considering an isothermal, or an isentropic, vapour flow, will be presented and discussed in the 
following part, whereas a new approach, considering the vapour phase at saturation state all along the heat pipe flow, will be also considered and compared to the two other approaches.

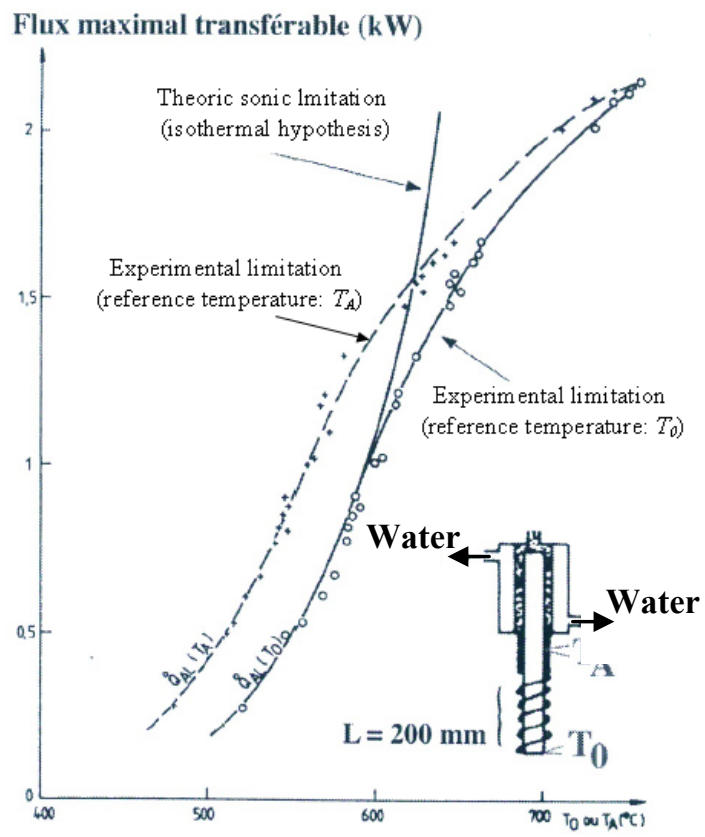

Fig. 5 Experimental results of sonic limitation for a sodium heat pipe (Gagneux, 1979).

\subsection{Review and discussion on existing approaches}

In Eq. (15), if the major difficulty lies in the values to be assigned to $h_{l v, a}, \rho_{v, a}$ and $T_{v, a}$ in the adiabatic zone, two classical approaches have been used involving isothermal and isentropic evolutions of the vapour flow between the evaporator and the adiabatic zone.

\section{Isothermal model}

Busse (1973) has studied an isothermal vapour flow $\left(T_{v, 0} / T_{v, 1}=1\right)$, considering the vapour as an ideal gas (see "Isothermal evolution" on Fig. 6). Using Eq. (15), with such a hypothesis, he found the following expression for the sonic limitation:

$Q_{\text {son, isot }}=\frac{\pi}{4} D_{v}^{2} h_{l v, a} \rho_{v, a} \sqrt{2 r_{g} T_{v, 0}}$

where " 0 " refers to the entrance to the evaporator, and $T_{v, a}=T_{v, 0}$. Assuming that the vapour is at saturation state at the entrance of the evaporator $\left(T_{v, 0}=T_{s a t}\left(P_{v, 0}\right)\right)$, that the evolution of the pressure along the evaporator is considered as isentropic ${ }^{2}$ and given by Eq. (18), and using the ideal gas law, one finally obtains:

$Q_{\text {son, }, \text { sot }}=\frac{\pi}{4} D_{v}^{2} h_{l v, a} \frac{P_{v, 0}}{(1+\gamma)} \sqrt{\frac{\gamma}{r_{g} T_{v, 0}}}$

\section{Isentropic model}

In isentropic models found in the literature (Deverall et al., 1970; Faghri, 1995; Peterson, 1994), the pressure evolution along the evaporator is given again by Eq. (18) and the vapour is still considered

\footnotetext{
${ }^{2}$ Considering the evolution of the pressure along the evaporator as isentropic permits to easily compare the models the ones to the others (i.e. for the same pressure drop).
}

as an ideal gas (see "isentropic evolution" on Fig. 6). For an isentropic flow, an energy balance for the whole evaporator gives:

$$
\frac{T_{v, 0}}{T_{v, 1}}=1+\frac{\gamma-1}{2} M a_{1}^{2}
$$

And, if $M a_{1}=1$, one has:

$\frac{T_{v, 0}}{T_{v, 1}}=\frac{\gamma+1}{2}$

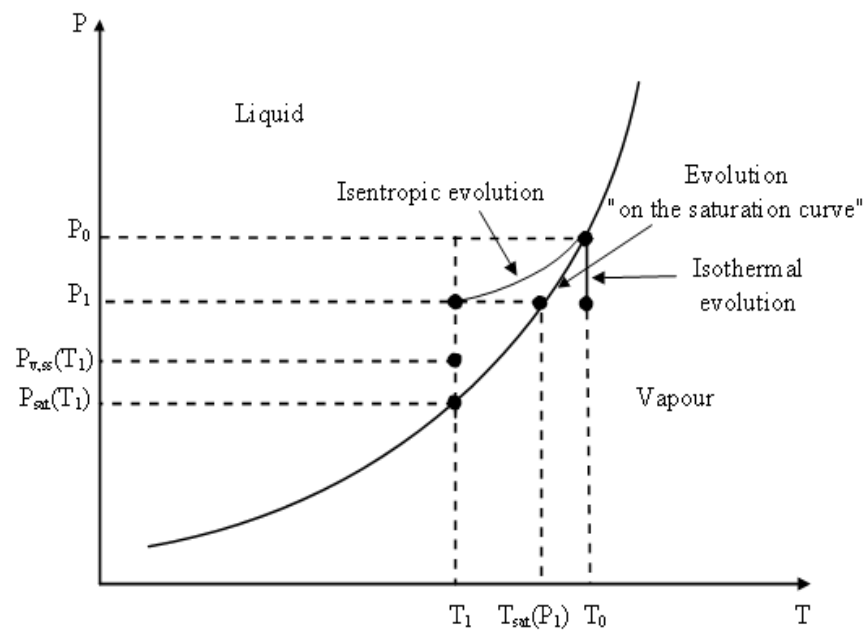

Fig. 6 Evolutions of the vapour flow along the saturation curve.

Using the general expression of Eq. (15) and the ideal gas law, the following expression can be obtained:

$Q_{\text {son, }, \text { isen }}=\frac{\pi}{4} D_{v}^{2} h_{l v, a} \frac{P_{v, a}}{\sqrt{T_{v, a}}} \sqrt{\frac{\gamma}{r_{g}}}$

Assuming that the vapour is at saturation state at the entrance of the evaporator, and using relations (18) and (22) for isentropic evolutions of both pressure and temperature, one finds:

$Q_{\text {son,isen }}=\frac{\pi}{4} D_{v}^{2} h_{l v, a} \frac{P_{v, 0}}{(1+\gamma) \sqrt{T_{v, 0}}} \sqrt{\frac{1+\gamma}{2}} \sqrt{\frac{\gamma}{r_{g}}}$

where "0" refers to the entrance of the evaporator at saturation state $\left(T_{v, 0}=T_{s a t}\left(P_{v, 0}\right)\right)$.

\section{Discussion on the two models}

The isothermal model's weakness derives from the fact that temperature evolution is assumed to be constant, even though it is generally admitted that along heat pipes, vapour temperature may evolve to some extent. Due to the thermophysical properties of the fluids, such evolution may modify the results obtained with the model, which has consequently been deemed somewhat unrealistic; that is one reason why alternative approaches have been developed.

Concerning the isentropic model, if the hypothesis of an ideal gas flow in a convergent nozzle is taken classically into account, the vapour phase cannot be considered at saturation state all along the heat pipe. Moreover, on the phase diagram, an isentropic evolution from point " 0 " in the saturation curve necessarily leads to a working point in the throat "1" situated in the liquid phase (cf. Fig. 6). As a result, the hypothesis of an isentropic flow with an operating point at saturation conditions likewise appears to be unrealistic, unless the vapour phase remains in a metastable state. 
Let us imagine the vapour phase to be at supersaturated state $\left(P_{v, s s}\right.$ on Fig. 6). Carey (1992) has shown that while a supersaturation state is conceivable for the vapour; a limitation exists beyond which droplet condensation can be triggered. Based on kinetic analysis of the droplets growing in the vapour phase and comparing the theory with experimental results, Carey obtained the following relation:

$\frac{P_{v, s s}\left(T_{v}\right)}{P_{s a t}\left(T_{v}\right)}=e^{\left(\frac{E^{*} \sqrt{-\ln J^{*}}}{2 \sqrt{E^{*}}+\left(-\ln J^{*}\right)^{3 / 2}}\right)}$

with $E^{*}=\frac{16 \pi \sigma^{3}}{3 k_{B} \rho_{l} r_{g}^{2} T_{v}^{3}}$ and $J^{*}=J \frac{M \rho_{l}}{N_{A}} \sqrt{\frac{\pi M}{2 \sigma N_{A}}}\left(\frac{r_{g} T_{v}}{P_{s a t}\left(T_{v}\right)}\right)$

where $J=10^{6} \mathrm{~m}^{-3} \mathrm{~s}^{-1}$ is the flux of droplets number per unit of volume, obtained through experimental results (Carey, 1992).

Eq. (5) determines the maximal supersaturation pressure $P_{v, s s}$ that can be admitted in the vapour phase for a given temperature $T_{v}$. Table 2 gathers numerical applications that locate the reference points of the vapour flow for Mach number equal to 1 at the end of the evaporator, taking an initial operating point $\left(P_{0}, T_{0}\right)$ at saturation state. The different characteristic points are represented on Fig. 6 , assuming that the vapour phase reaches the speed of sound at the throat: considering an isentropic evolution, the pressure at the throat $P_{1}$ always highly exceeds the maximal pressure of supersaturation $P_{v, s s}\left(T_{1}\right)$. As a result, appearance of droplets in the vapour phase inevitably occurs when the flow velocity at the end of the evaporator is sonic for an isentropic flow.

Table 2 Numerical applications for the evolution of the vapour phase at the evaporator.

\begin{tabular}{c|c|cc|c|c}
\hline \hline \multicolumn{1}{c|}{ Fluid } & \multicolumn{2}{|c|}{ Water } & Methanol & Sodium \\
\hline \hline $\begin{array}{c}\text { Initial point } \\
\text { "0" }\end{array}$ & $T_{0}$ & 40 & 60 & 0 & 427 \\
$P_{0}=P_{\text {sat }}\left(T_{0}\right)$ & 7370 & 19920 & 4050 & 95.1 \\
\hline \hline $\begin{array}{c}\text { Isentropic } \\
\text { evolution }\end{array}$ & $T_{1}$ (Eq. (22)) & -12 & 4.5 & -45.5 & 310 \\
$P_{1}$ (Eq. (18)) & 3100 & 8300 & 1690 & 40 \\
\hline \hline $\begin{array}{c}\text { Saturated } \\
\text { evolution }\end{array}$ & $T_{1}=T$ Tsat $\left(P_{1}\right)$ & 23 & 42 & -13 & 377 \\
\hline \hline & $P_{1}($ Eq. $(18))$ & - & 840 & 127 & 4 \\
\hline $\begin{array}{c}\text { Supersatur } \\
\text { a-tion state }\end{array}$ & $-\ln \left(J^{*}\right)$ & - & 55.8 & 52.4 & 42.0 \\
& $E^{*}$ & - & 103 & 30.7 & 3.05 \\
& $P_{v, s s}\left(T_{1}\right)$ & - & 3150 & 270 & 5 \\
\hline \hline
\end{tabular}

Temperatures in ${ }^{\circ} \mathrm{C}$, Pressures in $\mathrm{Pa}, J^{*}$ and $E^{*}$ dimensionless

Nevertheless, Levy (1968), considering a diphasic vapour flow model (vapour phase containing liquid droplets), gave results very close to those obtained by Busse's isothermal model (Eq. (20)). So, the use of this complex model seems to be somewhat inappropriate. This idea is supported by the lack of experimental data on the subject. Moreover, vapour in heat pipes is supposed to be totally dried and droplets can lead to deterioration of heat transport capacity of the heat pipe. Consequently, the vapour cannot remain at supersaturation state. This hypothesis is confirmed by the liquid/vapour interface which is necessarily close to the saturation state since it represents a zone for the condensation.

Thus, the vapour flow can no longer easily be considered neither isothermal nor as isentropic. Given this conclusion, vapour flow behaviour has been modified in the following part, and a new model has been built into the study.

\subsection{Sonic limitation considering saturated vapour flow}

In this case, it is assumed that vapour flow follows the saturation curve of the working fluid: it is supposed to be saturated and dry all along the heat pipe. From this hypothesis, a new expression of the sonic limitation may be proposed.

\section{New expression for the sonic limitation}

First, as already said, evolution of the thermophysical properties $P_{v}, T_{v}$ and $\rho_{v}$ on the saturation curve does not follow the ideal gas law. However, the gaz constant $r_{g}=P\left(T_{\text {sat }}\right) / \rho\left(T_{\text {sat }}\right) T_{\text {sat }}$ does not significantly vary for temperatures close to the triple point, and $r_{g}$ can be approximated, in a first approach, as a constant. Fig. 7 confronts the evolution of the ratio between $r_{g}$ and the specific gas constant $\left(r_{\text {specific }}=\right.$ $\left.R / M_{\text {vap }}\right)$, as a function of $\left(T-T_{T P}\right)$. The curves are drawn with regard to six fluids: water, methanol, ammonia, pentane, R134a and sodium. For the latter, the data close to the triple point are missing so the curves have been obtained for temperatures relatively far from the triple point. For methanol, water and pentane, the ratio $r_{g} / r_{\text {specific }}$ is particularly close to unity up until $\left(T-T_{T P}\right)$ reaches $100 \mathrm{~K}$, and decreases for greater temperature differences. For ammonia and R134a, the ratio remains close to unity up until $\left(T-T_{T P}\right)$ reaches $50 \mathrm{~K}$ and decreases for greater temperature differences. These are the fluids that have the highest density at these temperature levels. For sodium, one can suppose that in cases where $\left(T-T_{T P}\right)$ is inferior to $220 \mathrm{~K}$, the ratio remains close to 1 .

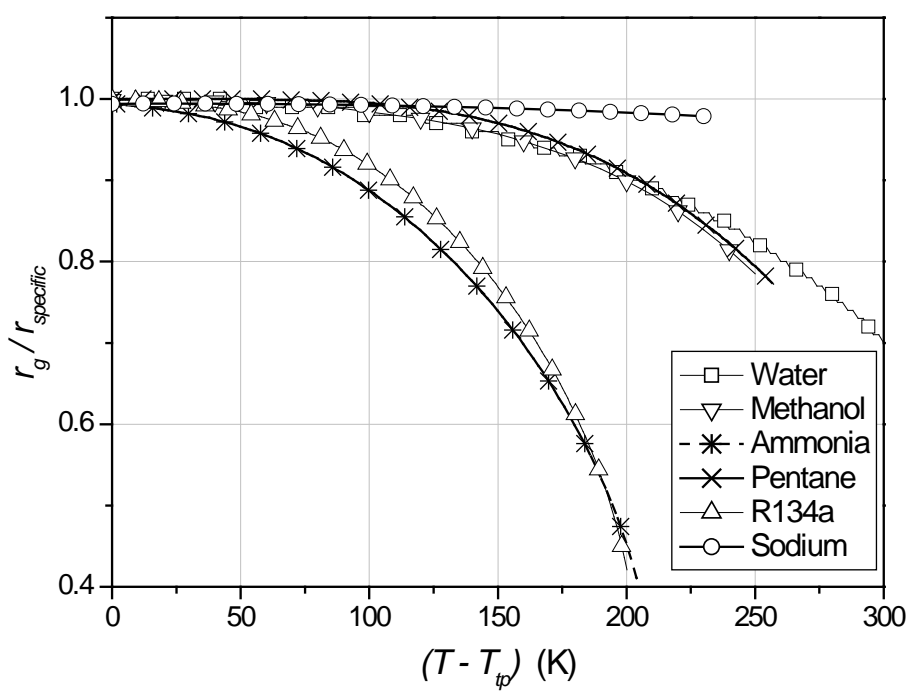

Fig. 7 Evolution of the ratio $r_{g} / r_{\text {specific }}$ as a function of $\left(T-T_{T P}\right)$ for six fluids.

For the six fluids, the approximation $r_{g} / r_{\text {specific }}=1$ is no longer valid when temperature increases on the saturation curve because of the pressure increase and on account of the irreversible phenomena associated with molecular shocks. If the vapour is assumed to be at saturation state all along the heat pipe, integrating the ClausiusClapeyron equation between sections " 1 " and " 0 " on Fig. 6, and using the ideal gas law $\left(r_{g}=P\left(T_{\text {sat }}\right) / \rho\left(T_{\text {sat }}\right) T_{\text {sat }}\right)$, Eq. (26) is obtained:

$\frac{P_{v, 0}}{P_{v, 1}}=e^{\frac{h_{l v}}{r_{g}}\left(\frac{1}{T_{v, 1}}-\frac{1}{T_{v, 0}}\right)}$

Starting from this, replacing “1” by “a”, using Eq. (15) and the previous approximation concerning $r_{g}$, one finally obtains: 


$$
Q_{\text {son }, \text { sat }}=\frac{\pi}{4} D_{v}^{2} h_{l v, a} \frac{P_{v, a}}{\sqrt{T_{v, a}}} \sqrt{\frac{\gamma}{r_{g}}}
$$

As in Eq. (20), to have the same pressure drop between the two evaporator's extremities for the three models (isothermal, isentropic and saturated vapour), the pressure evolution along the evaporator is given by Eq. (18). Combined with Eq. (26), one finally obtains:

$Q_{\text {son }, \text { sat }}=\frac{\pi}{4} D_{v}^{2} h_{l v, a} \frac{P_{v, 0}}{(1+\gamma)} \sqrt{\frac{\gamma}{r_{g} T_{v, 0}}} \frac{1}{\sqrt{1-r_{g} / h_{l v, a} T_{v, a} \ln (1+\gamma)}}$

where " 0 " and "a” are evaluated at the saturation state according to Eq. (26).

\section{Comparison of the different expressions for the sonic limitation}

The ratio between the sonic limitation in the case of isentropic flow $\left(Q_{\text {son,isen }}\right.$, Eq. (24)) and in the case of saturated vapour flow $\left(Q_{\text {son,sat }}\right.$, Eq. (28)) is given by the following relation:

$$
Q_{\text {son }, \text { sat }} / Q_{\text {son, }, \mathrm{sen}}=\sqrt{\frac{2}{(1+\gamma)\left(1-\frac{r_{g} T_{v, a}}{h_{l v, a}} \ln (1+\gamma)\right)}}
$$

In Eq. (29), the ratio $h_{l v, a, s a t} / h_{l v, a, \text { isen }}$ is set close to unity because, for temperatures close to the triple point, the latent heat of phase change does not significantly vary. Given this principle, Fig. 8 shows that the ratio $Q_{\text {son,sat }} / Q_{\text {son,isen }}$ is close to 1 for temperatures near the triple point: in general, it is included in the interval $[\mathbf{0 . 9} ; \mathbf{1}]$ for temperature differences lower than $100 \mathrm{~K}$, except for the sodium where it is lower than 0.9 , suggesting that this fluid is more sensitive to the isentropicity of the evolution than the others.

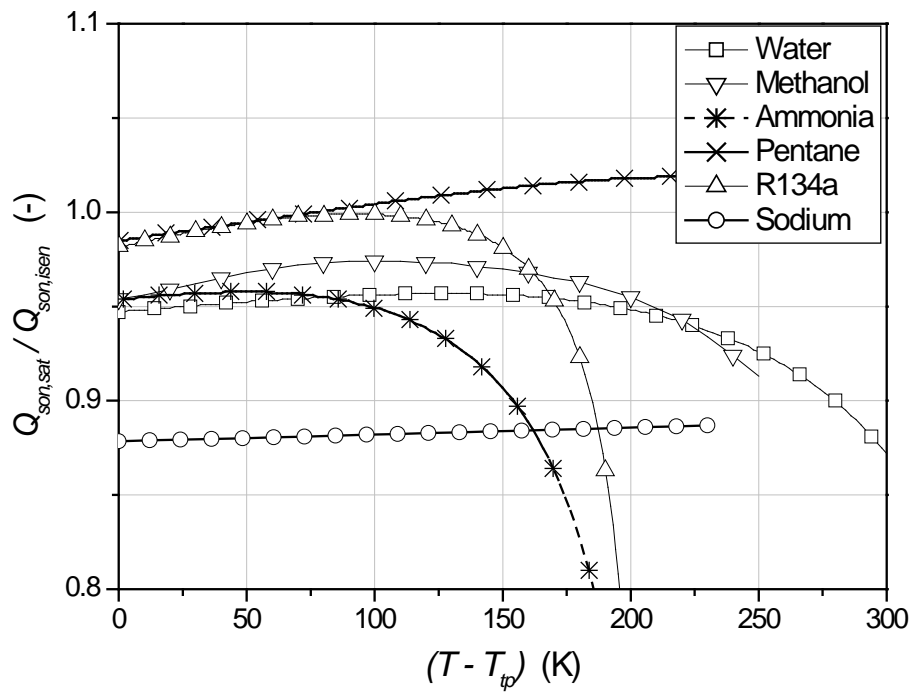

Fig. 8 Evolution of $Q_{\text {son,sat }} / Q_{\text {son,isen }}$ as a function of $\left(T-T_{T P}\right)$.

In the same way, the ratio between the sonic limitation in the case of isothermal flow $\left(Q_{\text {son,isot }}\right.$, Eq. (20)) and in the case of saturated vapour flow $\left(Q_{\text {son,sat }}\right.$, Eq. (28)) is given by:

$Q_{\text {son }, \text { sat }} / Q_{\text {son, isot }}=\left(1-r_{g} T_{v, a} \ln (1+\gamma) / h_{l v, a}\right)^{-1 / 2}$
The latter ration is typically included between $\mathbf{1}$ and $\mathbf{1 . 0 5}$ for temperatures near the triple point, as can be seen on Fig. 9. As a consequence, seeing the curves of Fig. 8 and 9, the sonic limitation determined with saturated vapour flow is situated between the isentropic model and the isothermal one.

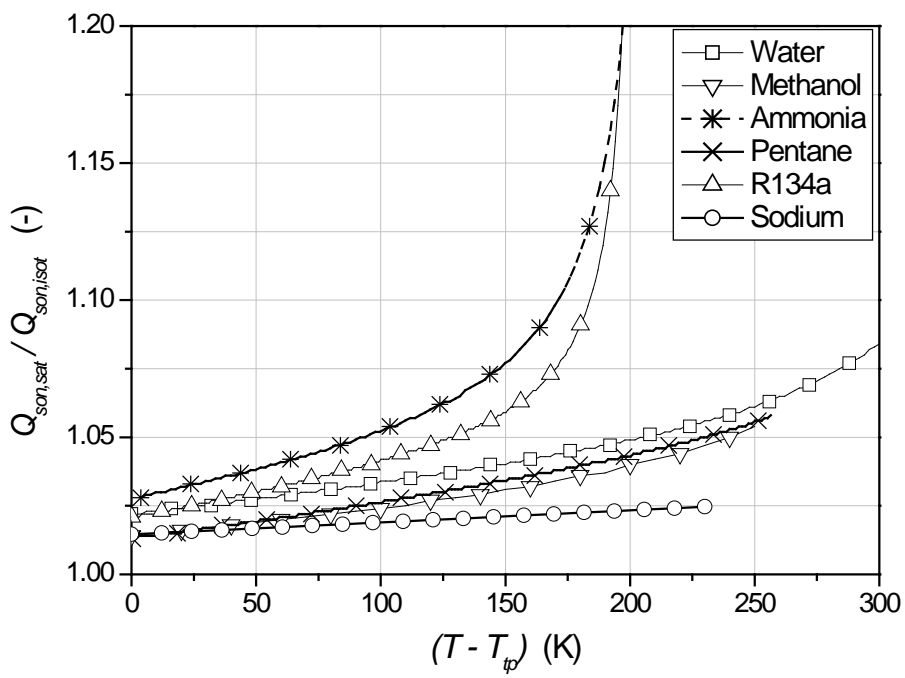

Fig. 9 Evolution of $Q_{\text {son,sat }} / Q_{\text {son,isot }}$ as a function of $\left(T-T_{T P}\right)$.

From these conclusions, it would be wise to compare the model results to the experimental ones obtained by Gagneux (1979) on a sodium heat pipe. Fig. 5 shows that the experimental results have a good agreement with Busse's isothermal model (Eq. (20)). Unfortunately, the measurements are not accurate enough to differentiate one of the three models to the others. Thus, for the instant, the approach considering a vapour evolution on the saturation curve has no major advantages compared to the isothermal model in view of the low accuracy obtained by experimental results; but, at least, the present model is physically quite more rigorous than the two other ones. In all cases, the actual sonic limitation, like that considering a saturation flow, is included between those considering the first two hypotheses, both bounding the vapour flow regime.

\subsection{Sonic limitation considering $2 \mathrm{D}$ vapour flow}

Busse (1973) has also treated the case of a two-dimensional, laminar and isothermal vapour flow. If the hypothesis of an isothermal flow is arguable, it still seems to be acceptable according to the previous section. In order to neglect radial pressure gradient, Busse has considered a symmetrical heat flux injection all around the heat pipe and a large evaporator length compared to the vapour diameter. Besides, the viscous losses are neglected. A momentum balance on the whole evaporator gives the relation:

$\bar{P}_{v, 0}-\bar{P}_{v, 1}=\overline{\rho_{v, 1} V_{v, 1}^{2}}$

Yet, the term $\overline{V_{v, 1}^{2}}$ cannot be directly evaluated with the data of the hydrodynamic problem because only the average velocity can be calculated as a function of the mass flow. Busse's study consisted in determining the ratio:

$A_{s}=\frac{\overline{V_{v, 1}^{2}}}{\bar{V}_{v, 1}^{2}}$

Considering a cosine velocity profile and a maximal velocity at the end of the evaporator, Busse found the value of $A_{s}$ equal to 1.11. If the 
vapour phase is considered at saturation state all along the evaporator, with Busse's results, one obtains:

$Q_{\text {son }}=\frac{\pi}{4 \sqrt{1.11}} D_{v}^{2} h_{l v, a} \rho_{v, a} \sqrt{\not r_{g} T_{v, a}}$

Eq. (33) gives values of the sonic limitation that are 5\% lower than those given by Eq. (28) corresponding to 1D saturated flow; the difference is small but not negligible.

Finally, DeMichele and Davis (1972) have performed numerical calculations for a two-dimensional laminar compressible flow considering the vapour as an ideal gas. The flow has been assumed to be isentropic, without viscous friction neither heat exchanges. They have obtained a large amount of results for several liquid metals. However, the latter results remain very close to the 1D model: the values obtained are superior of about $10 \%$ to experimental results, which is consistent with the present model (Eq. 28) which gives results only 5\% lower than isentropic model (Fig. 8).

\subsection{Sonic limitation considering viscous pressure losses}

Up to now, viscous pressure losses have not been taken into account in the different models of sonic limitation presented above. However, for heat pipes with long adiabatic zone, the pressure losses of the vapour flow become important due to the high values of its velocity (close to the speed of sound). Considering the latter pressure losses, the sonic limitation can be this time defined as the maximal transfer capability for a flow having a sonic velocity at the end of the adiabatic zone, i.e. where the vapour pressure is the lowest. Indeed when the vapour velocity is high enough, the inertial effects can cause an increase of the vapour pressure in the condensation zone (Romestant, 2000).

However, when the sonic limitation is determined using the temperature at the centre of the adiabatic zone as a reference (as it is the case in our model), the upstream vapour behaviour do not influence the sonic limitation. Only the pressure losses between the middle of the adiabatic zone and the condenser inlet are at the origin of a decrease of the sonic limitation. Consequently, using the adiabatic temperature as a reference, the present model is less sensitive to the viscous pressure losses.

Besides, Dobran (1989) led a study on the sonic limitation on a 1D flow of an ideal gas taking into account viscous losses both in evaporator and adiabatic zone. This model highlighted the apparition of a sonic velocity and the influence of the viscous losses for the determination of the sonic limitation. Yet, Dobran determined the sonic limitation using temperature at the evaporator inlet as a reference, which makes increase the difference with a model which does not take into account viscous losses. This last observation is an additional argument to choose the adiabatic temperature as a reference to determine the sonic limitation.

\subsection{Vapour radius determination: application to sonic limitation}

In the case of the sonic limitation, as seen before, the condenser does not take part in the calculations and the liquid film thickness is consequently assumed to be constant all along the adiabatic zone. Besides, neglecting the shear stresses causes only a little error on the sonic limitation.

\section{CONCLUSIONS}

In this paper, two heat pipes operating limitations, linked to the vapour flow, have been studied. The first one, viscous limitation, can be calculated assuming a laminar vapour flow. An analytical approach has been developed to obtain a first order solution. However, a numerical integration is possible to improve the precision of the calculations.
The second one, sonic limitation, has been defined showing that the classical approaches contain some hypotheses that are physically not acceptable even if the latter lead to results that remain close the one to the other and to experimental data. An original approach has been proposed: a more realistic hypothesis formulated on the hypothesis that the vapour flow remains at saturation state and leading to a simple expression of the sonic limitation.

Finally, let us notice that the transition between the two limits still represents an entire domain to investigate. Indeed, given the hypotheses of a pure viscous flow on one hand and of pure inertial flow, without viscous losses on the other hand, the existence of a transition zone where the physical phenomena are both present is predictable. Then, numerical calculations would be compulsory, this type of calculations only concerning a given type of heat pipe.

\section{NOMENCLATURE}

D Diameter (m)

g acceleration of gravity $\left(\mathrm{m} / \mathrm{s}^{2}\right)$

$h_{l v} \quad$ latent heat of phase change $(\mathrm{J} / \mathrm{kg})$

$L \quad$ heat pipe length (m)

$M \quad$ Molar mass (kg. mol $\left.{ }^{-1}\right)$

Ma Mach number (-)

$\dot{m} \quad$ mass flow $(\mathrm{kg} / \mathrm{s})$

$P \quad$ pression $(\mathrm{Pa})$

$q \quad$ heat flux density $\left(\mathrm{W} \cdot \mathrm{m}^{-2}\right)$

Q heat flux (W)

$r \quad$ radius $(\mathrm{m})$

$r_{g} \quad$ specific gas constant $(\mathrm{J} / \mathrm{kg} \cdot \mathrm{K})$

$S \quad$ surface $\left(\mathrm{m}^{2}\right)$

$T \quad$ temperature (K)

$U \quad$ velocity along $x$-axis $(\mathrm{m} / \mathrm{s})$

$\mathrm{V} \quad$ velocity $(\mathrm{m} / \mathrm{s})$

$y, z \quad$ coordinate $(\mathrm{m})$

\section{Greek Symbols}

$\alpha \quad$ geometric parameter $\left(\mathrm{m}^{3}\right)$

$\gamma \quad$ isentropic coefficient (-)

$\delta \quad$ film thickness (m)

$\mu \quad$ dynamic viscosity (Pa.s)

$\rho \quad$ density $\left(\mathrm{kg} / \mathrm{m}^{3}\right)$

$\tau \quad$ shear stess $\left(\mathrm{N} / \mathrm{m}^{2}\right)$

$\begin{array}{ll}\text { Subscripts } & \\ \text { a } & \text { adiabatic } \\ c & \text { condenser } \\ \text { cap } & \text { capillary } \\ e & \text { evaporator } \\ e b & \text { ebullition } \\ \text { eff } & \text { effective } \\ \text { ent } & \text { entrainement } \\ \text { isen } & \text { isentropic } \\ l & \text { liquid } \\ \text { sat } & \text { saturation } \\ \text { son } & \text { sonic } \\ \text { specific } & \text { specific } \\ \text { ss } & \text { supersaturation } \\ v & \text { vapour } \\ \text { vis } & \text { viscous } \\ w & \text { wall } \\ 0 & \text { entrance of the evaporator } \\ 1 & \text { inlet of the adiabatic zone }\end{array}$

\section{REFERENCES}

Bertossi, R. , Romestant, C., Ayel, V., Bertin, Y. 2010, “A new expression for sonic limitation in heat pipes", Proceedings of VIII 
Minsk International Seminar "Heat Pipes, Heat Pumps, Refrigerators, Power Sources", 405-412.

Busse, C.A, 1973, "Theory of the ultimate heat transfer limit of cylindrical heat pipes”, International Journal of Heat and Mass Transfer, 16, 169-185. http://dx.doi.org/10.1016/00179310(73)90260-3

http://dx.doi.org/10.1016/0017-9310(73)90260-3

Candel, S., 1990, Mécanique des Fluides, Dunod Université.

De Michele, D.W., Davis, M.V., 1972, "Vapor transport limits of liquid metal heat pipes”, Nuclear Technology, 15, 366-383.

Deverall, J.E., Kemme, J.E., Florschuetz, L.W., 1970, “Sonic limitation and startup problems of heat pipes," Los Alamos scientific laboratory, report $L A-4518$.

Dobran, F., 1989, "Supressions of the sonic heat transfer limit in high temperature heat pipes”, Journal of Heat Transfer, 111, 605-610. http://dx.doi.org/10.1115/1.3250725
Faghri, F.P., A., 1995, Heat pipe science and technology, Taylor et Francis.

Gagneux, P., 1979, Contribution à l'étude des caloducs à sodium, $\mathrm{PhD}$ Thesis, Université de Poitiers, LET, ENSMA.

Levy, E.K., 1968, "Theorical investigation of heat pipes operating at low vapor pressures”, ASME Aviation Space Conference, 15, 671-676.

NIST Scientific and Technical Database, National Institute of standards and Technology, http://webbook.nist.gov/chemistry/fluid/.

Peterson, G.P., 1994, Heat Pipes: modeling testing and applications, John Wiley \& Sons, Inc.

Romestant, C., 2000, Etudes théoriques et expérimentales de caloducs et de thermosiphons soumis à de fortes accélerations, $\mathrm{PhD}$ Thesis, Université de Poitiers, LET, ENSMA.

http://tel.archives-ouvertes.fr/docs/00/13/01/74/PDF/These$\underline{\text { Romestant.pdf }}$ 